\title{
Measurements for non-intrusive load monitoring through machine learning approaches
}

\author{
Giovanni Bucci ${ }^{1}$, Fabrizio Ciancetta ${ }^{1}$, Edoardo Fiorucci ${ }^{1}$, Simone Mari ${ }^{1}$, Andrea Fioravanti ${ }^{1}$ \\ ${ }^{1}$ University of L'Aquila, Piazzale E. Pontieri 1, 67100 L'Aquila, Italy
}

\section{ABSTRACT}

The topic of non-intrusive load monitoring (NILM) has seen a significant increase in research interest over the past decade, which has led to a significant increase in the performance of these systems. Nowadays, NILM systems are used in numerous applications, in particular by energy companies that provide users with an advanced management service of different consumption. These systems are mainly based on artificial intelligence algorithms that allow the disaggregation of energy by processing the absorbed power signal over more or less long time intervals (generally from fractions of an hour up to $24 \mathrm{~h}$ ). Less attention was paid to the search for solutions that allow non-intrusive monitoring of the load in (almost) real time, that is, systems that make it possible to determine the variations in loads in extremely short times (seconds or fractions of a second). This paper proposes possible approaches for non-intrusive load monitoring systems operating in real time, analysing them from the point of view of measurement. The measurement and postprocessing techniques used are illustrated and the results discussed. In addition, the work discusses the use of the results obtained to train machine learning algorithms that allow you to convert the measurement results into useful information for the user.

Section: RESEARCH PAPER

Keywords: Non-Intrusive Load Monitoring (NILM); energy management; Deep Learning (DL); Sweep Frequency Response Analysis (SFRA)

Citation: Giovanni Bucci, Fabrizio Ciancetta, Edoardo Fiorucci, Simone Mari, Andrea Fioravanti, Measurements for non-intrusive load monitoring through machine learning approaches, Acta IMEKO, vol. 10, no. 4, article 16, December 2021, identifier: IMEKO-ACTA-10 (2021)-04-16

Section Editors: Umberto Cesaro and Pasquale Arpaia, University of Naples Federico II, Italy

Received October 12, 2020; In final form December 6, 2021; Published December 2021

Copyright: This is an open-access article distributed under the terms of the Creative Commons Attribution 3.0 License, which permits unrestricted use, distribution, and reproduction in any medium, provided the original author and source are credited.

Corresponding author: Simone Mari, e-mail: simone.mari@graduate.univaq.it

\section{INTRODUCTION}

Nowadays, economic development has led to a steady increase in the demand for electricity and related advanced services. Companies are therefore moving towards the conversion of their traditional electrical systems into smart systems. One of the advantages that can be achieved is an efficient use of energy through the programming of loads and the awareness of consumption by users.

To this end it is necessary to know the information on the status and consumption of the various loads powered by the system. This can be achieved through intrusive monitoring, i.e. by installing individual sensors for each load. But also through non-intrusive monitoring, i.e. measuring the total power absorbed by the system and deducing the contributions of the individual loads from this, through the use of specific algorithms. In this second case, an extremely simple and compact measurement system is obtained, at the expense of greater complexity from the point of view of processing [1].
These non-intrusive monitoring systems, however, have proven effective on a wide range of applications, which go beyond energy management alone [2].

Non-intrusive load monitoring systems (NILMs) are used successfully in many applications, including demand response programs, where consumers can generate profits based on their flexibility [3], [4]. Other applications are those of Anomaly Detection, to detect malfunctions based on the profiles of power absorbed by individual loads [5] and of Condition-based maintenance, which has allowed the creation of monitoring systems capable of helping operators in maintenance planning [6], [7]. Finally, the Ambient Assisted Living is also very important, where the NILM system monitors the switching on and off of household appliances to infer the position and activities of people, detecting the space-time context and, therefore, the activities of daily life. of the subject [8]-[10].

The first NILM system was proposed by G. Hart in 1985 [11]. This algorithm was based on a detection of the edges in the aggregate power profile, followed by a clustering operation and subsequent matching based on the value of the absorbed power and on the on and off time. Clearly this approach, while being 
functional in certain situations, showed significant limitations as a multi-state appliance had to be managed as a set of distinct ON / OFF appliances. Conversely, continuously variable appliances and appliances with permanent consumption could not be detected correctly. It also included strong manual feature extraction requirements.

Subsequently other algorithms based on combinatorial optimization were proposed [12], whose main assumption is that each load can be in a given state (one of a reduced number $K$ ), in which each state is associated with a different energy consumption. The goal of the algorithm is to assign states to household appliances in such a way as to minimize the difference between the aggregate power reading and the sum of the energy consumption of the different loads.

In the last decade, thanks to the increase in available computing powers, attention has shifted to artificial intelligence algorithms, such as hidden-state Markov chains [13]-[15] and Deep Learning models [16]-[18]. In particular, the use of Deep Learning algorithms has overcome many of the limits that have characterized previous methods, thus allowing measurement systems to adapt to homes never analysed during the training phase. Furthermore, in terms of accuracy, systems based on convolutional neural networks have overcome other state-ofthe-art methods, such as those based on Factorial Hidden Markov Models [19].

However, the state of the art of these systems is represented almost exclusively by monitoring systems that process signals over time intervals of the order of hours, consequently the resulting feedback will not be in real time. Instead, it is often necessary to know in real time the status changes of the monitored loads. Non-intrusive load monitoring systems are therefore required, capable of recognizing the different powered devices based on signal processing, during intervals of seconds or fractions of seconds.

In this paper, two approaches for the recognition of electrical loads in real time will be presented. The first is a passive measurement system, based on the acquisition and processing of the current absorbed by the system. The second is an active measurement system, based on the measurement of the response to a variable frequency signal injected into the system.

\section{NILM SYSTEM BASED ON PASSIVE MEASUREMENTS}

A first attempt was made by creating a recognition system for electrical loads based on the analysis of the total absorbed current. A system of this type allows to obtain a low cost and galvanically isolated measuring system. In steady state conditions, the absorbed current does not provide sufficient information to characterize a wide range of different loads. This is because the waveform of the current absorbed by domestic loads hardly has a significant harmonic content. Therefore, the only considerations that can be made are based on the difference in amplitude. It was therefore decided to characterize the loads on the basis of their transient characteristics. Previous studies have tried to create NILM systems based on transient characteristics [20]-[23], but all have limited the analysis to a reduced number of loads and particular cases. In this study, on the other hand, tests were conducted on signals acquired from a test plant in which five commonly used household appliances were activated and deactivated, but above all the performance was also evaluated on the basis of the Building-Level fUlly-labeled public dataset for Electricity Disaggregation (BLUED) [24].
First, the rms value of the current is calculated by processing the acquired raw current with a sliding window technique, as follows:

$$
I_{\mathrm{rms}(k)}=\sqrt{\frac{1}{N} \sum_{n=k}^{k+(N-1)} i_{(n)}^{2}},
$$

where $k$ is the $k$-th measured current sample, $N$ is the number of samples per cycle, $i_{(n)}$ is the sampled signal, and $n$ is the summation index.

The rms value is then derived, and the resulting signal $I_{\mathrm{rms}(k)}^{\prime}$ is an impulsive signal in which each pulse corresponds to a change in state of one of the powered loads. An example is shown in Figure 1.

$$
I_{\mathrm{rms}(k)}^{\prime}=I_{\mathrm{rms}(k)}-I_{\mathrm{rms}(k-1)}
$$

The position of the pulse in the derived signal identifies the moment in which a certain event occurred.

In this way, the information relating to the steady state is filtered and only the information relating to the transients is kept. This impulsive signal is successively processed by the Short-time Fourier transform (STFT), through the following known transformation [25], [26]:

$$
\operatorname{STFT}_{(m, \omega)}=\sum_{n=-\infty}^{\infty} I_{\mathrm{rms}(n)}^{\prime} W_{(n-m)} e^{-j \omega n}
$$

Each change in the states of the powered loads is characterized and discriminated on the basis of the spectral content of the derived signal. The current is processed cyclically
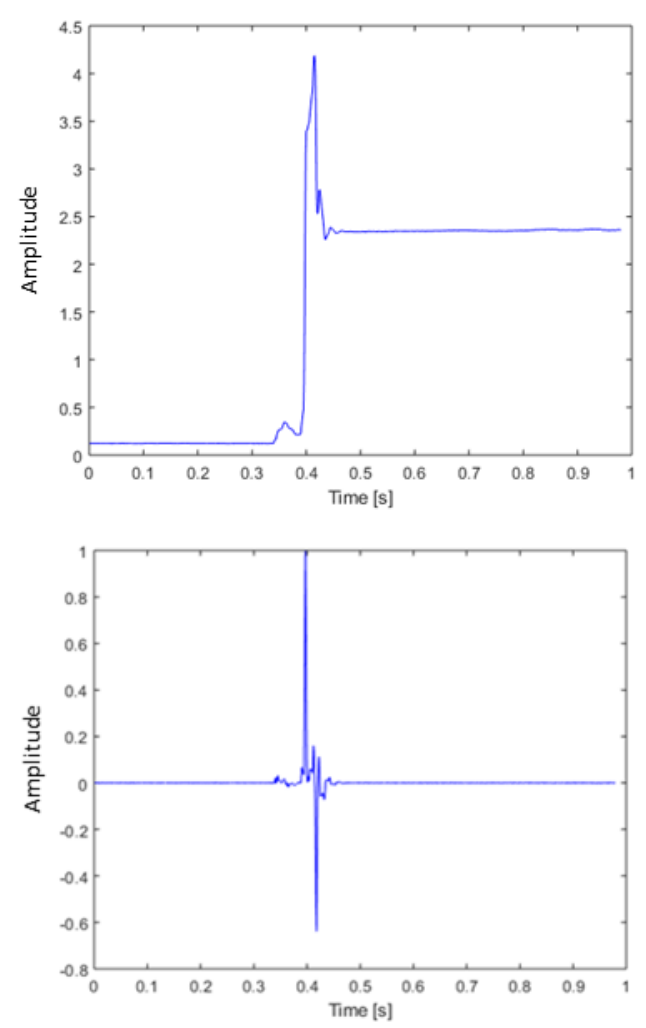

Figure 1. Variation with time of the rms current $I_{\mathrm{rms}(\mathrm{k})}$ (top) and its derivative $I_{\mathrm{rms}(\mathrm{k})}^{\prime}$ (bottom). 
at 1 second acquisition intervals, following the described procedure. Each acquisition slot is processed (to calculate rms and the derivative) by adopting an overlap of $500 \mathrm{~ms}$ to ensure correct analysis. It is also processed for transient events, which can be fragmented into two successive slots. The STFT is implemented by processing 10 -cycles $(200 \mathrm{~ms})$ windows with an overlap of $4 / 5$ of the processing window. This results in a spectrogram with 101 points in frequency at 26 different instants of time. To take into account the sign of the change (switching on, switching off or passing to a different consumption state), the spectrogram is multiplied by the sign of the cumulative sum, evaluated on the rms signal as follows:

$$
S_{N}=\sum_{n=1}^{N}\left(I_{\mathrm{rms}(n)}-I_{\mathrm{rms}(n-1)}\right)
$$

where $I_{\text {rms (n) }}$ is the rms value of the current described in (1), $N$ is the number of samples, and $S_{N}$ is the value of the cumulative sum. The final signal $S(i, j)$ can be obtained in the form of a $101 \times 26$ matrix, as follows:

$$
\begin{aligned}
S(i, j)= & \operatorname{STFT}_{(m, \omega)} \cdot \operatorname{sgn}\left(S_{N}\right) \\
= & \sum_{n=-\infty}^{\infty} I_{\mathrm{rms}(n)}^{\prime} w_{(n-m)} \mathrm{e}^{-j \omega n} \\
& \cdot \operatorname{sgn}\left(\sum_{n=1}^{N}\left(I_{\mathrm{rms}(n)}-I_{\mathrm{rms}(n-1)}\right)\right)
\end{aligned}
$$

An example of the spectrogram obtained from this procedure is shown in Figure 2.

This spectrogram is used as inputs to a neural network which provides a response every $500 \mathrm{~ms}$, indicating the presence or absence of events in the signal, and the type of device involved.

\subsection{The adopted Artificial Neural Network}

The deduction of the loads, starting from the spectrogram described above, is traced back to a multiclass classification problem, that is, a single unique label must be associated with each spectrogram. Analysing the current using such a small sliding window (1 second with 0.5 second overlap) makes it possible to state that within a single window there is no change of state of more than one load. Artificial Neural Networks (ANNs) are an example of algorithms that natively support multiclass classification problems.

In this work, a particular ANN type, namely, the convolutional neural network (CNN), is adopted [27] because of

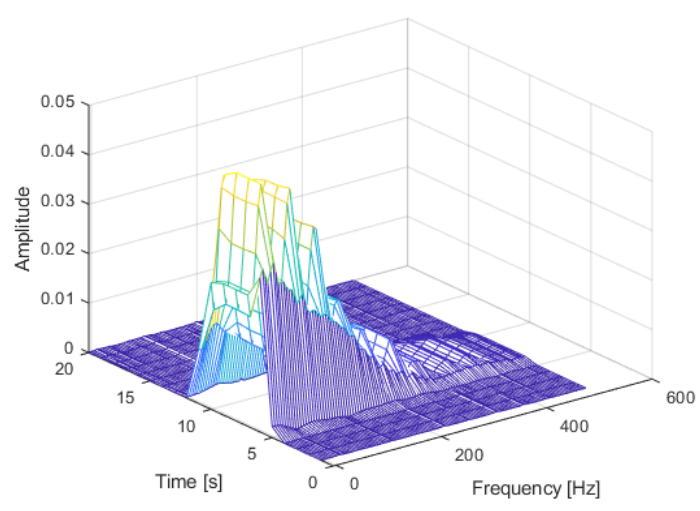

Figure 2. Spectrogram obtained during the switch ON of a microwave oven. its capability of processing complex inputs such as multidimensional arrays. More specifically, CNNs are designed to exploit the intrinsic properties of some two-dimensional data structures, in which there is a correlation between spatially close elements (local connectivity).

The proposed system [28] includes different layers: an input level (for signal loading), three groups of layers, each of which consisting of convolution, Relu, and max pooling layers (for feature extraction from the input), and a group of flatten, fully connected, and softmax layers, which uses data from convolution layers to generate the output.

\subsection{The proposed system setup}

The proposed measurement system uses an Agilent U2542A data acquisition module with a 16-bit resolution. The current signal was acquired using a TA SCT-013 current transducer and the sampling frequency was set to $10 \mathrm{kHz}$. The CNN network was implemented on a desktop computer (based on the Windows $10 \times 64$ operating system) using the open-source Python 3.7 from Anaconda.

Tests were conducted on signals acquired directly from a real system, in order to have flexibility both as regards the sampling frequency and for the generation of multiple events. Other tests were conducted on signals belonging to the public BLUED dataset, which features 34 different types of devices.

The proposed measurement system was installed on a test system, designed to generate electrical loads made by domestic users, as part of the research project "Non-intrusive infrastructures for monitoring loads in residential users". The system, which is located in the Electrical Engineering Laboratory of the University of L'Aquila (I), allows the generation of electrical loads in a single or simultaneous way. These loads correspond to the loads generated by the most common household appliances and are integrated in a structure similar to that of a residential building to reproduce the real problems of conditioning and measurement of the signals.

\subsection{The obtained results}

The performance of the NILM system was assessed by conducting acquisitions, during which various loads were turned $\mathrm{ON}$ and OFF for a total of over 519 events.

Next, BLUED, a public dataset on residential electricity usage, was used. This dataset includes voltage and current measurements for a single-family house in the United States, sampled at $12 \mathrm{kHz}$ for an entire week.

Regarding NILM systems, no standard and consolidated techniques can be found in the literature to evaluate the performance of event detectors. Since the purpose of a NILM system is to disaggregate consumption for each of the devices in question, their performances were analysed to verify the achievement of these objectives, which in summary are correct identification and classification of the events. These parameters were obtained using the number of true positive (TP), false positive (FP), true negative (TN), and false negative (FN). In addition, the accuracy was assessed as follows:

$$
\text { Accuracy } \%=\frac{\text { Correct matches }}{\text { Total possible matches }} 100 \%
$$

The obtained results are summarized in Table 1. 
Table 1. Scores achieved with the acquired signal and the BLUED dataset.

\begin{tabular}{lcc}
\hline & Acquired signal & BLUED dataset \\
\hline Precision & 0.981 & 0.998 \\
Recall & 0.998 & 0.998 \\
F1-Score & 0.989 & 0.998 \\
Accuracy \% & $98.0 \%$ & $87.9 \%$ \\
\hline
\end{tabular}

\section{NILM SYSTEM BASED ON ACTIVE MEASUREMENTS}

Subsequently, we tried to recognize the loads powered by an electrical system through the Sweep Frequency Response Analysis (SFRA). The SFRA is a non-destructive diagnostic technique that detects the displacement and deformation of windings, among other mechanical and electrical failures, in power and distribution transformers. SFRA proceeds by applying a sinusoidal voltage signal of constant amplitude and variable frequency between one terminal of the bipole under test and ground. The response is measured between the other bipole terminal and ground. Both input and output signals are acquired and processed. The obtained result is the Transfer Function (TF) of the bipole over a wide frequency range. A failure is detected when a change in the TF is observed.

The possibility of using these traces to identify which devices are powered at the time of measurement was evaluated [29]. The basic idea is to detect a change in the load, starting from the change in the measured TF. For this purpose, a variable frequency sinusoidal signal is applied between the terminal of the power phase conductor and ground, by means of the instrumentation shown in Figure 3, then both the applied input signal and the output signal between the neutral conductor terminal and ground are measured and processed. The test instrument generates a sinusoidal input signal of constant amplitude (a few volt) and frequency variable in the range between $10 \mathrm{kHz}$ and $1.5 \mathrm{MHz}$. The results obtained on the electrical system are analysed on a temporal basis, comparing them with those previously obtained on the same system. The measurement techniques follow the IEC 60076-18 standard [30], which regulates the test execution methods, the characteristics of the instruments used, the connection methods and the analysis of the results. Figure 4 shows the signatures obtained for the different types of loads.

Tests were also conducted in order to evaluate the ability to discriminate through these traces different loads when they are

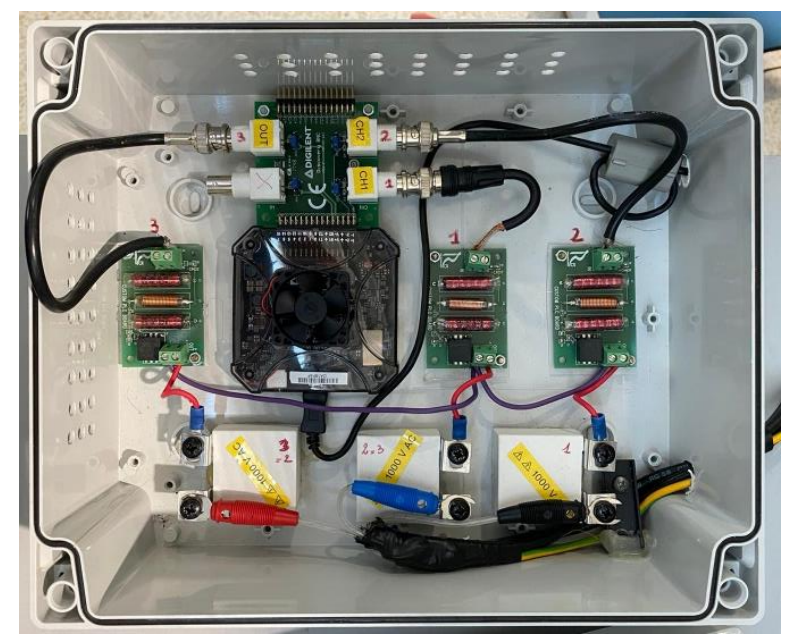

Figure 3. Instrumentation used for the SFRA.

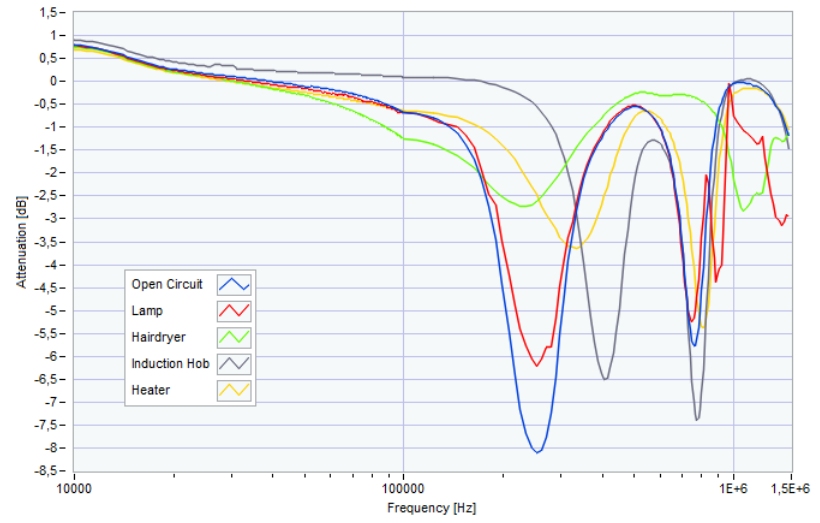

Figure 4. SFRA tests of different household appliances.

powered simultaneously. From Figure 5 it is possible to see the possibility to discriminate, through these traces, if the heater is powered individually or in combination with other loads.

\subsection{The Machine Learning approaches}

In order to translate these traces into useful information for the users, the problem was formulated as a multi-label classification problem. This is a variant of the classification problem, where multiple labels (or multiple classes) may be assigned to each instance. Multi-label classification is a generalization of multiclass classification, which is the singlelabel problem of categorizing instances into precisely one of more than two classes. In the multi-label problem, there is no constraint on how many of the classes the instance can be assigned to. The problem was initially addressed with an ANN [31] similar to the one described in the previous section, with good results. However, a limitation of the ANNs is the large amount of training data required, which makes it difficult to apply them in real cases. An attempt was therefore made to use another machine learning algorithm, the Support Vector Machine (SVM).

SVM is one of the most popular artificial intelligence algorithms and is a supervised learning algorithm used primarily for solving classification problems. Unlike generic classification algorithms that discriminate on the basis of characteristics common to each class, SVM focuses on the samples that are most similar to each other but belonging to different classes, which are therefore the most difficult samples to discriminate.

On the basis of these samples, the algorithm constructs an optimal hyperplane capable of separating them, and which can then be used to discriminate the new samples. These samples are

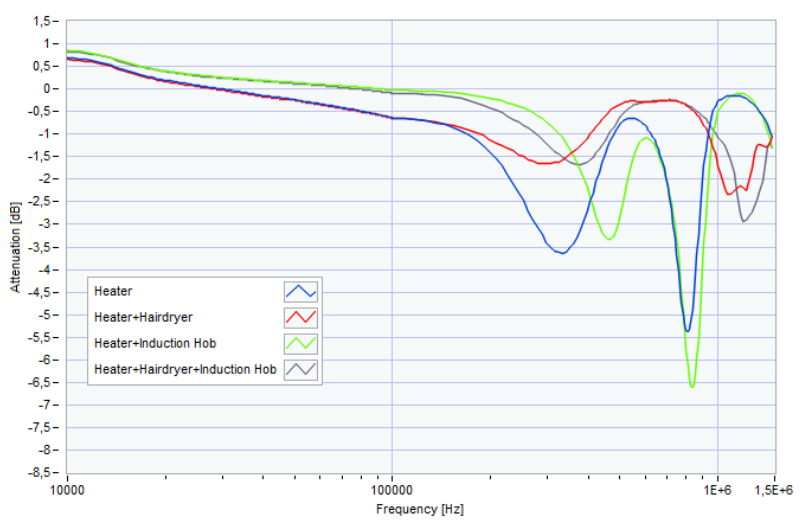

Figure 5. SFRA tests with simultaneous loads powered. 
called support vectors, because they are the only samples that support model creation, while all other samples are useless.

In a two-dimensional case, where the examples to be classified are defined by only two characteristics, the optimal hyperplane is reduced to a straight line as shown in Figure 6. The algorithm searches for the line that maximizes the margin between the two examples indicated as support vectors. If it is not possible to separate the classes with a straight line, as in the case of nonlinear classification problems, the algorithm uses the kernel trick [32]. In particular, a polynomial nucleus was chosen for this work, thus examining not only the given characteristics of the input samples to determine their similarity, but also their combinations.

In the case of the proposed NILM system, the problem is obviously not two-dimensional. In fact, the SFRA measuring system returns an array of 320 points, which represent the transfer functions corresponding to the different frequency values. Therefore, the inputs of the SVM is 320, and consequently also the size of the problem is 320 . To solve the problem, to identify which devices are powered starting from the result of the SFRA measurement, four SVM classifiers are used, each of which performs a binary classification, identifying the presence or absence of the device associated with it.

\subsection{The obtained results}

Unlike the NILM system based on passive measurements, in this case there is no public dataset that has the necessary characteristics, i. e. there is no public dataset of measurements obtained through the SFRA technique. Therefore, the performance evaluation was made solely on the basis of our acquired measurements. As for the previously described system, also in this case the same test parameters were used. The proposed algorithm was subjected to different scenarios, each for
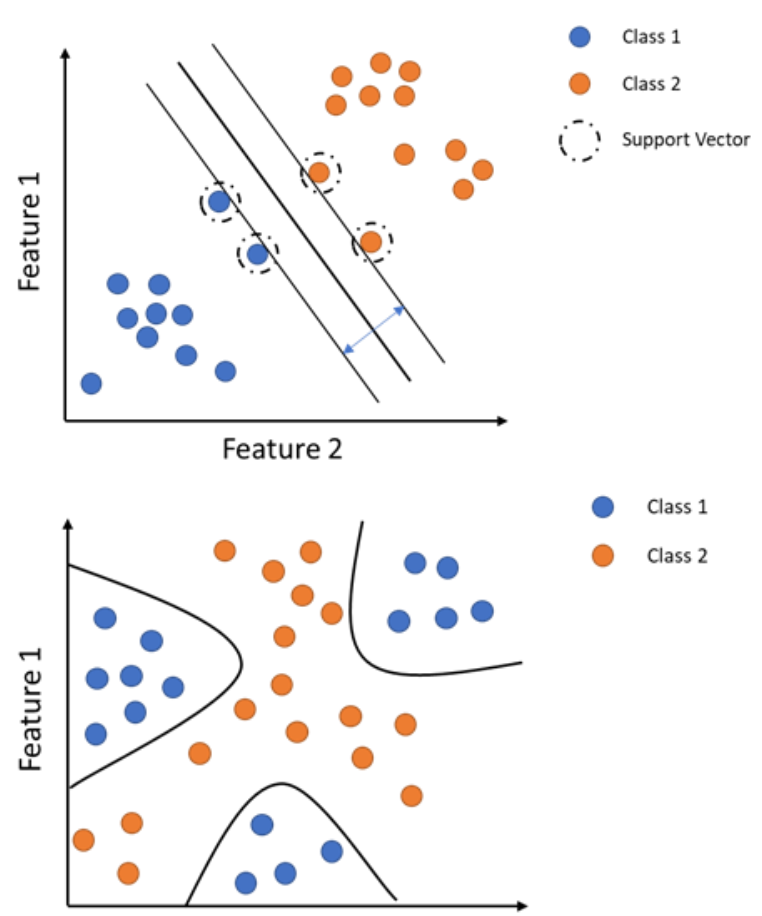

Class 1

Class 2

Feature 2

Figure 6. Representation of a linear classification problem (top) and a nonlinear classification problem (bottom) in which the samples are defined by only two features.
Table 2. Achieved scores with the SVM.

\begin{tabular}{ccccc}
\hline & $\begin{array}{c}\text { SVM } \\
\text { Lamp }\end{array}$ & $\begin{array}{c}\text { SVM } \\
\text { Hairdryer }\end{array}$ & $\begin{array}{c}\text { SVM } \\
\text { Induction Hob }\end{array}$ & $\begin{array}{c}\text { SVM } \\
\text { Heater }\end{array}$ \\
\hline TP & 42 & 200 & 200 & 200 \\
FP & 0 & 0 & 0 & 2 \\
TN & 400 & 250 & 250 & 248 \\
FN & 8 & 0 & 0 & 0 \\
Precision & 1 & 1 & 1 & 0.99 \\
Recall & 0.84 & 1 & 1 & 1 \\
F1-Score & 0.91 & 1 & 1 & 0.99 \\
\hline
\end{tabular}

a certain number of tests, in which the different appliances were powered individually or simultaneously.

Since, as already explained above, each appliance has an associated SVM algorithm that reveals its presence or not, the performance of the four algorithms were assessed individually. To allow a comparison with the algorithms developed by other researchers, precision, recall, F1-Score and accuracy during classification were evaluated [33]. The results are shown in Table 2.

As far as the ANN is concerned, the evaluation measures for a multiclass, hence single-label classification problem, are generally different from those for the multiple label. In singlelabel classification we can use simple metrics such as precision, recall, and accuracy [34]. However, in the multi-label classification, an incorrect classification is no longer a real error, as a forecast containing a subset of the actual classes is certainly better than a forecast that does not, i.e. correctly predicting two of the four labels is better than foresee the absence of labels. To evaluate the performance of a multi-label classifier we have to calculate the average of the classes. There are two different methods of doing this called micro-averaging and macroaveraging [35]. The current is processed cyclically at 1 second acquisition the metric independently for each class and then take the average hence treating all classes equally, whereas the microaverage will aggregate the contributions of all classes to compute the average metric. In a multi-label classification setup, microaverage is preferable if there is a suspicion that there may be a class imbalance (i.e. the possibility of having many more examples of a class than other classes). In the cases under examination, this problem does not exist as the examples used for training and testing are sufficiently uniform, so micro-average and macro-average can both be considered reliable.

Precision $_{\text {micro-averaging }}=\frac{\sum_{n=1}^{N} T P_{n}}{\sum_{n=1}^{N} T P_{n}+F P_{n}}$

Recall $_{\text {micro-averaging }}=\frac{\sum_{n=1}^{N} T P_{n}}{\sum_{n=1}^{N} T P_{n}+F N_{n}}$

F1 - score micro-averaging

$=\frac{2 \times \text { Precision }_{\text {micro-averaging }} \times \text { Recall }_{\text {micro-averaging }}}{\text { Precision }_{\text {micro-averaging }}+\text { Recall }_{\text {micro-averaging }}}$

Precision $_{\text {macro-averaging }}=\frac{\sum_{n=1}^{N} \text { Precision }_{n}}{N}$

Recall $_{\text {macro-averaging }}=\frac{\sum_{n=1}^{N} \operatorname{Recall}_{n}}{N}$

F1 - score macro-averaging

$=\frac{2 \times \text { Precision }_{\text {macro-averaging }} \times \text { Recall }_{\text {macro-averaging }}}{\text { Precision }_{\text {macro-averaging }}+\text { Recall }_{\text {macro-averaging }}}$ 
Table 3. Comparison between SVM and ANN.

\begin{tabular}{l|cc|cc}
\hline & \multicolumn{2}{|c|}{ SVM } & \multicolumn{2}{c}{ ANN } \\
\hline & $\begin{array}{c}\text { Micro- } \\
\text { averaging }\end{array}$ & $\begin{array}{c}\text { Macro- } \\
\text { averaging }\end{array}$ & $\begin{array}{c}\text { Micro- } \\
\text { averaging }\end{array}$ & $\begin{array}{c}\text { Macro- } \\
\text { averaging }\end{array}$ \\
\hline Precision & 0.99 & 0.99 & 0.94 & 0.91 \\
Recall & 0.98 & 0.96 & 0.99 & 0.99 \\
F1-Score & 0.98 & 0.97 & 0.96 & 0.95 \\
\hline
\end{tabular}

Table 3 shows the scores achieved according to the two criteria both with the ANN and with the SVM.

\section{CONCLUSIONS AND FINAL REMARKS}

In this paper a brief introduction of the state of the art of NILM systems has been presented. Two different types of systems for real-time identification of electrical loads, based on different measurement techniques, were then presented. Both systems were proven excellent identification performance.

More in detail, the first system, based on the spectrogram analysis of the effective current through the $\mathrm{CNN}$, has been proven excellent performance in correspondence with both the acquired measurements and those available in the BLUED dataset, reaching F1-Score respectively equal to at 0.989 and 0.998 and Accuracy $\%$ respectively equal to $98.0 \%$ and $87.9 \%$.

The greatest difficulty encountered in the classification phase with the BLUED dataset is attributable to the significantly greater number of devices that the network is required to recognize, compared to those used for the acquired measurements. Furthermore, the value obtained for the F1-score is higher than that obtained with other systems using the same dataset, as those proposed in [36] (0.915) and [37] (0.932).

Traditional NILM systems perform the loads classification based on the analysis of quantities also related to voltage (e.g. analysis in the P-Q or V-I plan [38]). The proposed system has the advantage of measuring only the overall current in a house. As a result, the complexity of the processing system is reduced. Another advantage is that the measuring system can be implemented with a galvanically isolated at low-cost system, using a clamp current transducer.

The second proposed system, based on the trace analysis provided by the SFRA, also proved excellent performance. The traces were initially processed through an artificial neural network similar to that used for the previous system, reaching F1-Score of 0.96. In order to reduce the number of training examples needed, it was decided to use a Support Vector Machine. Despite a significant reduction in the examples needed for training (from over 2000 to 90), the F1-Score achieved with this second machine learning structure was even higher than that obtained with the artificial neural network.

A system of this type is particularly interesting as it allows the creation of a plug-in solution that can be installed in any domestic, industrial or commercial environment. Furthermore, the detection technique takes into account the physical characteristics of household appliances and the resulting transfer function. Consequently, the identification of multi-state or continuously variable appliances is simplified, compared to processing time-varying signals such as real power, current, etc.

\section{REFERENCES}

[1] G. Bucci, F. Ciancetta, E. Fiorucci and S. Mari, Load identification system for residential applications based on the NILM technique,
2020 IEEE International Instrumentation and Measurement Technology Conference (I2MTC), 25-28 May 2020, pp. 1-6. DOI: $\underline{10.1109 / \mathrm{I} 2 \mathrm{MTC} \text { T3012.2020.9128599 }}$

[2] G. Bucci, F. Ciancetta, E. Fiorucci, S. Mari, A. Fioravanti, State of art overview of Non-Intrusive Load Monitoring applications in smart grids, Measurement: Sensors 18(2021), art. no. 100145. DOI: $10.1016 / \mathrm{j} . \mathrm{measen} .2021 .100145$

[3] A. Lucas, L. Jansen, N. Andreadou, E. Kotsakis, M. Masera, Load flexibility forecast for DR using non-intrusive load monitoring in the residential sector, Energies 12(14) (2019), art. no. 2725.

DOI: $10.3390 /$ en12142725

[4] W. Schneider, F. Campello de Souza, Non-intrusive load monitoring for smart grids, 2018. Technical report. DELL EMC.

[5] H. Rashid, P. Singh, V. Stankovic, L. Stankovic, Can non-intrusive load monitoring be used for identifying an appliance's anomalous behaviour?, Applied Energy 238 (2019), pp. 796-805.

DOI: $10.1016 /$ j.apenergy.2019.01.061

[6] D. Green, T. Kane, S. Kidwell, P. Lindahl, J. Donnal, S. Leeb, NILM dashboard: Actionable feedback for condition-based maintenance, IEEE Instrumentation \& Measurement Magazine 23(5) (2020), pp. 3-10.

DOI: $10.1109 /$ MIM.2020.9153467

[7] A. Aboulian et al., NILM dashboard: A power system monitor for electromechanical equipment diagnostics, IEEE Transactions on Industrial Informatics 15(3) (2019), pp. 1405-1414.

DOI: $10.1109 /$ TII.2018.2843770

[8] C. Belley, S. Gaboury, B. Bouchard, A. Bouzouane, An efficient and inexpensive method for activity recognition within a smart home based on load signatures of appliances, Pervasive and Mobile Computing 12 (2014), pp. 58-78.

DOI: $10.1016 /$ i.pmcj.2013.02.002

[9] N. Noury, M. Berenguer, H. Teyssier, M. Bouzid,M. Giordani, Building an index of activity of inhabitants from their activity on the residential electrical power line, IEEE Transactions on Information Technology in Biomedicine 15(5) (2011), pp. 758766.

DOI: $10.1109 /$ TITB.2011.2138149

[10] X. Zhang, T. Kato, T. Matsuyama, Learning a context-aware personal model of appliance usage patterns in smart home,2014 IEEE Innovative Smart Grid Technologies - Asia (ISGT ASIA), Kuala Lumpur, Malaysia, 20-23 May 2014, pp. 73-78. DOI: 10.1109/ISGT-Asia.2014.6873767

[11] G. Hart. 1985. Prototype nonintrusive appliance load monitor. In MIT Energy Laboratory Technical Report, and Electric Power Research Institute Technical Report.

[12] G. W. Hart. 1992. Nonintrusive appliance load monitoring. Proc. IEEE 80, 12 (Dec. 1992), 1870-1891.

DOI: $10.1109 / 5.192069$

[13] J Zico Kolter and Matthew J Johnson. 2011. REDD: A public data set for energy disaggregation research.

[14] Oliver Parson, Siddhartha Ghosh, Mark Weal, Alex Rogers, Nonintrusive load monitoring using prior models of general appliance types, 2012 Twenty-Sixth AAAI Conference on Artificial Intelligence, Toronto, Canada, 22-26 July 2012.

[15] Mingjun Zhong, Nigel Goddard, and Charles Sutton. 2014. Signal Aggregate Constraints in Additive Factorial HMMs, with Application to Energy Disaggregation. In Advances in Neural Information Processing Systems 27, Z. Ghahramani, M. Welling, C. Cortes, N. D. Lawrence, and K. Q. Weinberger (Eds.). Curran Associates, Inc., 3590-3598.

[16] J. Kelly, W. Knottenbelt, Neural NILM: Deep neural networks applied to energy disaggregation, 2nd ACM International Conference on Embedded Systems for Energy-Efficient Built Environments (BuildSys '15). Association for Computing Machinery, New York, NY, USA, pp. 55-64. DOI: $10.1145 / 2821650.2821672$

[17] Z. Jia, L. Yang, Z. Zhang, H. Liu, F. Kong, Sequence to point learning based on bidirectional dilated residual network for nonintrusive load monitoring, International Journal of Electrical Power \& Energy Systems 129(2021), art. no. 106837. 
DOI: $10.1016 /$ i.ijepes.2021.106837

[18] G. Bucci, F. Ciancetta, E. Fiorucci, S. Mari, A. Fioravanti, Multistate appliances identification through a NILM system based on convolutional neural network, 2021 IEEE Instrumentation and Measurement Technology Conference I2MTC 2021, 17-21 May 2021.

DOI: $10.1109 /$ I2MTC50364.2021.9460038

[19] C. Zhang, M. Zhong, Z. Wang, N. Goddard, C. Sutton, Sequenceto-point learning with neural networks for non-intrusive load monitoring, AAAI Conf. Artif. Intell., New Orleans, LA, USA, Feb. 2018, pp. 2604-2611.

[20] H. Chang, K. Chen, Y. Tsai and W. Lee, A new measurement method for power signatures of nonintrusive demand monitoring and load identification, IEEE Transactions on Industry Applications 48(2) (2012), pp. 764-771. DOI: $10.1109 /$ TIA.2011.2180497

[21] Y. Lin, M. Tsai, Development of an improved time-frequency analysis-based nonintrusive load monitor for load demand Identification, IEEE Transactions on Instrumentation and Measurement 63(6) (2014), pp. 1470-1483. DOI: $\underline{10.1109 / T I M .2013 .2289700}$

[22] H. Chang, K. Lian, Y. Su, W. Lee, Power-spectrum-based wavelet transform for nonintrusive demand monitoring and load identification, IEEE Transactions on Industry Applications 50(3) (2014), pp. 2081-2089.

DOI: $10.1109 /$ TIA.2013.2283318

[23] S. B. Leeb, S. R. Shaw, J. L. Kirtley, Transient event detection in spectral envelope estimates for nonintrusive load monitoring, IEEE Transactions on Power Delivery 10(3) (1995), pp. 12001210. DOI: $10.1109 / 61.400897$

[24] K. Anderson, A. Ocneanu, D. Benitez, D. Carlson, A. Rowe, M. Bergés, BLUED: a fully labeled public dataset for event-based non-intrusive load monitoring research, 2nd KDD Workshop on Data Mining Applications in Sustainability (SustKDD), Beijing, China, Aug. 2012, pp. 1-5.

[25] W. Yuegang, J. Shao, X. Hongtao, Non-stationary signals processing based on STFT, 8th International Conference on Electronic Measurement and Instruments, Xi'an, 2007, pp. 3-3013-304.

DOI: 10.1109/ICEMI.2007.4350914

[26] S. Zhang, D. Yu, S. Sheng, A discrete STFT processor for realtime spectrum analysis, APCCAS 2006 - 2006 IEEE Asia Pacific Conference on Circuits and Systems, Singapore, 4-7 Dec. 2006, pp. 1943-1946.

DOI: $10.1109 /$ APCCAS.2006.342241

[27] S. Albawi, T. A. Mohammed, S. Al-Zawi, Understanding of a convolutional neural network, International Conference on Engineering and Technology (ICET), Antalya, Turkey, 21-23 Aug. 2017, pp. 1-6.
DOI: $10.1109 /$ ICEngTechnol.2017.8308186

[28] F. Ciancetta, G. Bucci, E. Fiorucci, S. Mari, A. Fioravanti, A new convolutional neural network-based system for NILM applications, IEEE Transactions on Instrumentation and Measurement 70 (2021), art no. 1501112. DOI: $10.1109 /$ TIM.2020.3035193

[29] A. Fioravanti, A. Prudenzi, G. Bucci, E. Fiorucci, F. Ciancetta, S. Mari, Non-intrusive electrical load identification through an online SFRA based approach, 2020 International Symposium on Power Electronics, Electrical Drives, Automation and Motion (SPEEDAM), Sorrento (Italy), 24-26 June 2020, pp. 694-698. DOI: $10.1109 /$ SPEEDAM48782.2020.9161856

[30] IEC 60076-18:2012 Power transformers - Part 18: Measurement of frequency response

[31] G. Bucci, F. Ciancetta, E. Fiorucci, S. Mari, A. Fioravanti, Deep Learning applied to SFRA Results: a Preliminary Study, 7th International Conference on Computing and Artificial Intelligence ICCAI 2021, Tianjin, China, 23-26 April 2021, pp. 302-307 DOI: $10.1145 / 3467707.3467753$

[32] T. Hofmann et al., Kernel methods in machine learning, Ann. Statist. 36(3) (2008), pp. 1171-1220.

DOI: $10.1214 / 009053607000000677$

[33] G. Bucci, F. Ciancetta, E. Fiorucci, S. Mari, A. Fioravanti, A nonintrusive load identification system based on frequency response analysis, 2021 IEEE International Workshop on Metrology for Industry 4.0 \& IoT (MetroInd4.0\&IoT), 7-9 June 2021, pp. 254258.

DOI: 10.1109/MetroInd4.0IoT51437.2021.9488472

[34] S Makonin, F Popowich, Nonintrusive load monitoring (NILM) performance evaluation, Energy Efficiency 8(4) (2014), pp. 809814. DOI: $\underline{10.1007 / \mathrm{s} 12053-014-9306-2}$

[35] O. Koyejo, N. Natarajan, P. K. Ravikumar, I. S. Dhillon, Consistent multilabel classification, in Proc. NIPS, 2015, pp. 3321-3329.

[36] M. A.Peng, H. Lee, Energy disaggregation of overlapping home appliance consumptions using a cluster splitting approach, Sustainable Cities and Society 43 (2018), pp. 487-494. DOI: $10.1016 /$ i.scs.2018.08.020

[37] K. Jain, S. S. Ahmed, P. Sundaramoorthy, R. Thiruvengadam, V. Vijayaraghavan, Current peak based device classification in NILM on a low-cost embedded platform using extra-trees, IEEE MIT Undergraduate Research Technology Conference (URTC), Cambridge, MA, -5 Nov. 2017, pp. 1-4. DOI: $10.1109 /$ URTC.2017.8284200

[38] T. Hassan, F. Javed, N. Arshad, An empirical investigation of V-I trajectory-based load signatures for non-intrusive load monitoring, IEEE Transactions on Smart Grid 5(2) (2014), pp. 870-878. DOI: $10.1109 /$ PESGM.2014.6938824 